РЕГИОНАЛЬНАЯ И МУНИЦИПАЛЬНАЯ ЭКОНОМИКА REGIONAL AND MUNICIPAL ECONOMY

УДК $332.1(470+571)$

DOI 10.18413/2687-0932-2020-47-2-233-243

\title{
ПЕРСПЕКТИВНЫЕ ЭКОНОМИЧЕСКИЕ СПЕЦИАЛИЗАЦИИ ДЛЯ РОССИЙСКИХ РЕГИОНОВ В СТРАТЕГИИ ПРОСТРАНСТВЕННОГО РАЗВИТИЯ: КЛУБЫ КОНВЕРГЕНЦИИ
}

\section{PERSPECTIVE ECONOMIC SPECIALIZATIONS FOR THE RUSSIAN REGIONS IN THE STRATEGY OF SPATIAL DEVELOPMENT: CONVERGENCE CLUBS}

\author{
В.И. Блануца \\ V.I. Blanutsa \\ Институт географии им. В.Б. Сочавы СО РАН, \\ Россия, 664033, г. Иркутск, ул. Улан-Баторская, 1 \\ V.B. Sochava Institute of Geography of Siberian Branch of RAS, \\ 1 Ulan-Batorskaya St, Irkutsk, 664033, Russia \\ E-mail: blanutsa@list.ru
}

\begin{abstract}
Аннотация
В «Стратегии пространственного развития Российской Федерации на период до 2025 года» приведены перспективные экономические специализации для каждого региона и сформулирована цель в виде сокращения межрегиональных различий. В статье проанализировано соответствие между декларируемой целью и распределением экономических специализаций по российским регионам. Методологической основой исследования стала концепция экономической конвергенции, согласно которой траектории развития множества регионов сходятся к одному (абсолютная конвергенция) или нескольким (относительная конвергенция) уровням. Предложена мера сходства регионов по сочетанию перспективных экономических специализаций. Расчеты показали, что гипотеза абсолютной конвергенции российских регионов по перспективным специализациям не подтвердилась. Сделан вывод о несоответствии между распределением специализаций по регионам и целью пространственного развития. Для проверки гипотезы относительной (клубной) конвергенции использовался кластерный анализ. Российские регионы по сходству специализаций объединены в шесть клубов конвергенции. Первый клуб можно считать экономически устойчивым по множеству специализаций. Остальные пять клубов имеют мало специализаций и в условиях экономического кризиса могут быть неустойчивыми. В стратегии выявлен ряд концептуальных и эмпирических ошибок. Для обнаружения и исправления других ошибок предложено провести независимую экспертизу стратегии.
\end{abstract}

\begin{abstract}
The "Spatial Development Strategy of the Russian Federation for the Period until 2025" contains promising economic specializations for each region and formulates a goal in the form of reducing inter-regional differences. The article analyzes the correspondence between the declared goal and the distribution of economic specializations in the Russian regions. The methodological basis of the study was the concept of economic convergence, according to which the development paths of many regions converge to one (absolute convergence) or several (relative convergence) levels. A measure of regional similarity in the combination of promising economic specializations is proposed. Calculations showed that the hypothesis of absolute convergence of the Russian regions for promising specializations was not confirmed. It is concluded that there is no correspondence between the distribution of specializations by regions and the goal of spatial
\end{abstract}


development. To test the hypothesis of relative (club) convergence, cluster analysis was used. By the similarity of specializations, the Russian regions are combined into six convergence clubs. The first club can be considered economically sustainable in many specialties. The remaining five clubs have few specializations and may be unstable in the context of the economic crisis. According to the strategy of spatial development in Russia, a certain center will be formed from the regions of the first club and the periphery from the regions of other clubs. This will result in a specific territorial structure in which peripheral highly specialized regions will be located (except for one region) along the state border of the Russian Federation. The strategy identified a number of conceptual and empirical errors. To detect and correct other errors, it was proposed to conduct an independent examination of the strategy.

Ключевые слова: региональная экономика, пространственное развитие, стратегическое планирование, экономическая специализация, регион, кластерный анализ, клубная конвергенция, Российская Федерация.

Keywords: regional economy, spatial development, strategic planning, economic specialization, region, cluster analysis, club convergence, Russian Federation.

\section{Введение}

В феврале 2019 г. была утверждена «Стратегия пространственного развития Российской Федерации на период до 2025 года» (далее - Стратегия) [Распоряжение ..., 2019]. Данный документ вызвал множество критических замечаний в научном сообществе [Бухвальд, Кольчугина, 2019; Котов, 2019; Кузнецова, 2019; Лексин, 2019; Минакир, 2019]. В трактовку пространственного развития включено несколько новаций для постсоветского стратегического планирования. Одна из них - «перспективные экономические специализации» для каждого субъекта (региона) Российской Федерации. Однако в Стратегии не приведено обоснование таких специализаций, что существенно отличает рассматриваемый документ от генеральной схемы размещения и развития производительных сил СССР [Смирнова, 2014]. К настоящему времени опубликовано две статьи по анализу перспективных экономических специализаций - по общим вопросам [Иванов, Бухвальд, 2019] и ситуации в Свердловской области [Смирнова, Аверина, 2019].

При стратегическом планировании обычно происходит выбор между «пространственной справедливостью» и «экономической эффективностью» [Humer, 2018; Мельникова, 2018]. В первом случае ресурсы направляются на поддержку отстающих регионов, что обосновывается концепцией «экономической конвергенции» [Barro, Sala-iMartin, 1992; Islam, 2003]. Во втором случае происходит наоборот - поддерживаются передовые регионы в соответствии с концепцией «полюсов роста» [Perroux, 1950; Parr, 1999]. Целью Стратегии является «обеспечение устойчивого и сбалансированного пространственного развития Российской Федерации, направленного на сокращение межрегиональных различий в уровне и качестве жизни населения, ускорение темпов экономического роста и технологического развития, а также на обеспечение национальной безопасности страны» [Распоряжение ..., 2019, с. 7-8], что будет достигаться в том числе «за счет повышения конкурентоспособности экономик субъектов Российской Федерации путем обеспечения условий для развития производства товаров и услуг в отраслях перспективных экономических специализаций субъектов Российской Федерации» [Распоряжение ..., 2019, c. 8]. При декларировании сокращения различий между регионами («пространственная справедливость») в Стратегии основное внимание (68 из 114 стр.) уделено перечням перспективных экономических специализаций и перспективных центров экономического роста. Эти перечни нацелены на «экономическую эффективность». Если центры роста укладываются только в концепцию «полюсов роста», то по экономическим специализациям не исключена возможность конвергенции регионов и установления «пространственной справедливости». Однако ранее в мировой науке не предпринимались попытки 
проанализировать перспективные экономические специализации регионов с позиции концепции конвергенции экономического развития.

Под экономической конвергенцией регионов в наиболее общем виде понимается схождение траекторий развития регионов к некоторому уровню. Применительно к различным социально-экономическим показателям регионального развития этот уровень принимает разные значения. Например, в Европейском союзе регион считается слабо экономически развитым и может рассчитывать на помощь при валовом региональном продукте на душу населения ниже $75 \%$ от среднего уровня по союзу [Воронов, 2014]. В данном случае конвергенция достигается при выходе всех регионов на средний уровень по заданному показателю с допуском его уменьшения на 25 \%. Если все регионы достигли установленного уровня, то имеет место абсолютная (общая) конвергенция. При схождении траекторий развития регионов к нескольким иерархическим уровням формируется относительная (клубная) конвергенция. Если вместо схождения наблюдается расхождение траекторий развития регионов, то возникает дивергенция. Конвергенция может проходить с разной скоростью [Marelli, Parisi, Signorelli, 2019] и на определенных этапах развития замещаться дивергенцией [Napolitano, Pietroluongo, Kounetas, 2018; Kinfemichael, Morshed, 2019].

Формирование клубов конвергенции происходит благодаря сходству исходных структурных характеристик регионов [Galor, 1996], близости регионов для передачи неявного знания [Audretsch, Feldman, 1996] и некоторых других особенностей [Tian et al., 2016; von Lyncker, Thoennessen, 2017; Celbis, de Crombrugghe, 2018; Barrios, Flores, Angeles, 2019; Marelli, Parisi, Signorelli, 2019]. Наличие клубной конвергенции устанавливается не только по душевому доходу или производительности труда [Napolitano, Pietroluongo, Kounetas, 2018; Kinfemichael, Morshed, 2019], но и по душевому потреблению энергии [Ivanovski, Churchill, Smyth, 2018], количеству патентов на миллион жителей [Barrios, Flores, Angeles, 2019], моделям безработицы [Monfort, Ordonez, Sala, 2018] и другим социально-экономическим показателям и структурам. В некоторых случаях экономическая конвергенция понимается очень широко, о чем свидетельствуют экономические исследования по сближению культур [Tarabar, 2019] и эмиссий $\mathrm{CO}_{2}$ [Rios, Gianmoena, 2018]. С учетом всех отмеченных особенностей в статье будут проверены гипотезы о наличии абсолютной и относительной конвергенции российских регионов по сочетанию перспективных экономических специализаций, зафиксированных в Стратегии.

\section{Объекты и методы исследования}

В 2019 г. в Российской Федерации было 85 субъектов (регионов), из них для 84 в Стратегии приведены перспективные экономические специализации. Можно только предположить, что разработчики Стратегии не стали приводить перспективные специализации для Москвы, так как экономическое развитие столицы не нуждается в федеральной поддержке. Специализации были сформированы как группы видов, взятых из Общероссийского классификатора видов экономической деятельности. Всего в Стратегии представлено 34 специализации. Наиболее часто в российских регионах перспективными считаются такие специализации, как «производство прочих готовых изделий» (в 83 регионах), «Туризм» (72), «растениеводство и животноводство» (70), «производство машин и оборудования, не включенных в другие группировки» (68) и «производство пищевых продуктов» (68), а наименее часто - «обеспечение электрической энергией, газом и паром» (1), «ремонт и монтаж машин и оборудования (ремонт и техническое обслуживание судов и лодок)» (2) и «производство табачных изделий» (5). Наибольшее количество перспективных экономических специализаций имеют Республика Татарстан, Нижегородская и Ростовская области (по 27 специализаций), а наименьшее - Ненецкий автономный округ (4).

Для сравнения российских регионов между собой по сочетанию перспективных экономических специализаций предлагается использовать простую меру сходства: 


$$
U_{i j}=\frac{2 N_{i j}}{N_{i}+N_{j}}
$$

где $U_{i j}$ - мера сходства между регионами $i$ и $j ; N_{i j}$ - количество одинаковых специализаций, встречающихся в регионах $i$ и $j ; N_{i}, N_{j}$ - количество всех специализаций в регионах $i$ и $j$. Если в двух регионах встречаются одни и те же специализации, то имеет место полное сходство $\left(U_{i j}=1\right)$, а если в сравниваемых регионах наблюдаются разные наборы специализаций, то получается отсутствие какого-либо сходства $\left(U_{i j}=0\right)$. Что касается установления уровня конвергенции, то это значение должно отражать допустимое отклонение всех регионов от региона с максимальным набором специализаций. При этом обычно учитываются промежуточные и конечные цели регионального развития, исходная экономическая дифференциация регионов и мировой опыт изучения конвергенции [Islam, 2003; Monfort, Ordonez, Sala, 2018; Kinfemichael, Morshed, 2019]. В нашем случае мировой опыт отсутствует, исходные (2019 г.) наборы специализаций для регионов не приведены и конечная (2025 г.) цель диверсификации экономических специализаций не сформулирована. Для проверки гипотезы об абсолютной конвергенции в условиях такой неопределенности целесообразно оперировать максимально допустимым отклонением в $50 \%$, что соответствует нижнему порогу согласования региональных социально-экономических структур [Блануца, 2018б]. Тогда если сходство всех регионов с регионами-лидерами укладывается в это отклонение $\left(U_{i j} \geq 0,500\right)$, то имеет место абсолютная экономическая конвергенция; в противном случае клубная конвергенция.

При относительной конвергенции в первый клуб входят регионы, имеющие максимально допустимое отклонение от регионов-лидеров $\left(U_{i j} \geq 0,500\right)$, во второй и последующие клубы - остальные регионы. Если вне первого клуба остался один регион, то он формирует второй клуб, который становится последним клубом. Наличие нескольких регионов за пределами допустимого отклонения приводит к необходимости распределения этих регионов по клубам. Обычно для этого используется кластерный анализ [Barrios, Flores, Angeles, 2019; Gozgor, Lau, Lu, 2019]. Кластеризацию регионов на основе $U_{i j}$ целесообразно (по данному классу мер сходства) проводить с помощью алгоритма автоматического социально-экономического районирования [Блануца, 2018б]. В нашем случае принята следующая последовательность действий: из симметричной матрицы $\left\{U_{i j}\right\}$ удаляются значения первого клуба регионов; среди оставшихся значений выбирается максимальная мера сходства $\left(\max U_{i j}\right)$ при условии $U_{i j} \geq 0,500$ (невыполнение этого условия означает, что каждый регион представляет собой отдельный клуб) и образуется первый кластер; вводится шаг кластеризации $\left(\Delta U_{i j}=0,001\right)$ и среди оставшихся мер сходства опять выбирается максимальное значение, что позволяет сформировать второй кластер; отдельный регион может войти в ранее созданный кластер на шаге $k$ только в случае приемлемого сходства $\left(U_{i j} \geq \max U_{i j}-k \Delta U_{i j}\right)$ со всеми регионами этого кластера (аналогичным образом происходит объединение нескольких кластеров); процедура выполняется до тех пор, пока все регионы не будут распределены по кластерам и останутся только значения $U_{i j}<0,500$ (регион, не объединившийся с другими регионами, является клубом).

\section{Результаты и их обсуждение}

В симметричной матрице мер сходства $\left\{U_{i j}\right\}$, имеющей размер $84 \times 84$, наибольшее совпадение перспективных специализаций достигнуто между Республикой Татарстан и Нижегородской областью $\left(U_{i j}=0,963\right)$, а наименьшее - между Чувашской Республикой и Магаданской областью $(0,080)$, Республикой Саха (Якутия) и Псковской областью $(0,080)$, Магаданской и Ульяновской областями $(0,080)$. Относительно трех ведущих регионов (Республика Татарстан, Нижегородская и Ростовская области) зафиксировано 17 регионов вне допустимого сходства $\left(U_{i j}<0,500\right)$. Таким образом, не подтвердилась гипотеза абсолютной 
экономической конвергенции российских регионов по заданному параметру и принятым ограничениям. Отсюда следует, что цель Стратегии («сокращение межрегиональных различий») даже при очень низком уровне допустимого сходства $\left(U_{i j} \geq 0,500\right)$ не может быть достигнута к 2025 г. по перспективным экономическим специализациям субъектов Российской Федерации.

По допустимому сходству с тремя регионами-лидерами в первый клуб конвергенции вошло 67 регионов. Среди оставшихся регионов наибольшее сходство было между Ненецким и Ямало-Ненецким автономными округами $\left(\max U_{i j}=0,800\right)$, а наименьшее - между Республикой Тыва и Сахалинской областью $(0,143)$. На 21-м шаге произошло второе объединение (Забайкальский край и Еврейская автономная область; $U_{i j}=0,778$ ), а на 49-м шаге - третье объединение (Камчатский край и Сахалинская область; $U_{i j}=0,750$ ). Всего было выполнено 12 объединений, позволивших сформировать 5 кластеров (клубов конвергенции). В итоге 84 региона России были распределены по 6 клубам следующим образом.

Клуб 1: все регионы, кроме перечисленных ниже (32 из 34 специализаций).

Клуб 2: Республика Калмыкия, Амурская и Костромская области (специализации, встречающиеся во всех регионах клуба: «производство прочих готовых изделий», «производство химических веществ и химических продуктов», «растениеводство и животноводство» и «туризм»).

Клуб 3: Республики Карелия, Коми и Саха (Якутия) («добыча полезных ископаемых», «лесоводство и лесозаготовки», «обработка древесины и производство изделий из дерева, кроме мебели», «производство бумаги и бумажных изделий», «производство прочих готовых изделий» и «туризм»).

Клуб 4: Камчатский край, Магаданская, Мурманская и Сахалинская области, Чукотский автономный округ («добыча полезных ископаемых», «производство прочих готовых изделий», «транспортировка и хранение» и «рыболовство и рыбоводство»).

Клуб 5: Республики Алтай и Тыва, Забайкальский край и Еврейская автономная область («лесоводство и лесозаготовки», «производство прочих готовых изделий» и «растениеводство и животноводство»).

Клуб 6: Ненецкий и Ямало-Ненецкий автономные округа («добыча полезных ископаемых», «производство прочих готовых изделий», «деятельность в области информации и связи» и «транспортировка и хранение»).

Выделение клубов конвергенции производилось по перспективным экономическим специализациям, приведенным в Стратегии, но распределение некоторых специализаций по регионам вызывает ряд вопросов. Отметим только некоторые из них. Почему «производство прочих готовых изделий» встречается во всех регионах, кроме Псковской области (это ошибка или имеется какой-то особый смысл в данном исключении)? Почему «обеспечение электрической энергией, газом и паром» перспективно только для Амурской области, в то время как экспорт электроэнергии характерен и для некоторых других регионов (и в более существенном объеме)? Если в каком-то регионе (например, на юго-западе России) возникнет необходимость в «ремонте и техническом обслуживании судов и лодок», то транспортные средства придется доставлять в Республику Саха (Якутия) и Сахалинскую область (по Стратегии подобный ремонт возможен только в этих двух регионах)? Почему в регионах с небольшим количеством экономических специализаций и уникальными природными комплексами не является перспективным такой драйвер регионального роста, как «туризм» (такой специализации нет в Республике Тыва, Забайкальском крае, Магаданской области, Ненецком, Чукотском и Ямало-Ненецком автономных округах)?

Следует также отметить в Стратегии непонятную формулировку «неперспективная экономическая специализация, критически важная для экономики». Если специализация так важна для региона, то почему она не является перспективной? «Неперспективные специализации» в нашем исследовании не учитывались из-за их неопределенного статуса. Такие специализации присутствуют в 33 регионах, а больше всего их в Республике Саха (Якутия), Тверской области и Ямало-Ненецком автономном округе (по 4). Среди 34 специализаций чаще 
всего «неперспективными» были «растениеводство и животноводство» (в 9 регионах) и «обработка древесины и производство изделий из дерева, кроме мебели» (в 8). При этом в Вологодской области «производство прочей неметаллической минеральной продукции» оказалось одновременно как в перспективных, так и неперспективных специализациях, а в Калининградской области в подобной ситуации оказалось «производство прочих готовых изделий». Неопределенность, внесенная разработчиками Стратегии в распределение перспективных и неперспективных специализаций по регионам, дополняется отсутствием критериев выделения перспективных экономических специализаций. Эмпирические исследования современной ситуации в отдельных отраслях указывают на иную территориальную структуру специализации. Например, при изучении специализации российских регионов на телекоммуникационных услугах было установлено, что формирование двух крупных телекоммуникационных хабов (в Москве и Санкт-Петербурге) лишило остальные регионы Европейской России необходимости специализации на таких услугах, вытеснив функцию специализации на периферию - Урал и Сибирь - и создав окраинный эффект (в Калининградской области и трех регионах Дальнего Востока) [Блануца, 2017].

Формулировка специализаций по Стратегии никак не отражает запланированный переход к цифровой экономике [Распоряжение ..., 2017]. Из всех специализаций только «производство компьютеров, электронных и оптических изделий» и «деятельность в области информации и связи» в какой-то мере можно отнести к будущей цифровой экономике. Обе специализации прописаны для 45 регионов. Можно ли отсюда сделать заключение, что в оставшихся 39 регионах не планируется развитие цифровой экономики? При этом ни один регион из клубов 2-6 не имеет двух отмеченных специализаций вместе. О перспективной «цифровой» специализации регионов и общероссийской территориальной структуре такой специализации ничего не сказано в анализируемом документе, хотя уже имеются первые попытки идентификации подобных структур [Блануца, 2018а]. Переход к цифровой экономике для некоторых отстающих регионов может послужить дополнительным драйвером экономического роста. Однако в данном случае потребуются специфические экономические специализации, например, на создании «территориальной цифровой платформы» [Блануца, 2019] и формировании «платформенной экономики» [Montalban, Frigant, Jullien, 2019; Spulber, 2019].

Принятый в Стратегии подход к агрегированию видов экономической деятельности в перспективные отрасли не учитывает современные концепции экономической специализации. К примеру, концепцию «умной специализации» [Feder, 2018; Foray, 2018; Ranga, 2018; Balland et al., 2019; Lopes, Ferreira, Farinha, 2019], согласно которой отыскиваются внутренние (эндогенные) факторы экономического роста и осуществляется концентрация ресурсов на уникальных компетенциях региона. Для столь разнообразной страны как Российская Федерация на основе этой концепции, например, можно было бы уточнить расплывчатую специализацию «туризм». Тогда в каждом регионе была бы своя, специфическая компетенция в области туристической деятельности, создающая конкурентные преимущества [Borsekova, Vanova, Vitalisova, 2017]. Именно обоснование и развитие «умной» специализации будет способствовать развитию периферийных регионов, а не обобщенно-непонятное «производство прочих готовых изделий».

Обозначение перспективных экономических специализаций подразумевает, что правительство будет поддерживать инвестиционные проекты в регионах именно по этим отраслям, хотя это не столь очевидно [Иванов, Бухвальд, 2019]. Такая стратегия не исключает местную инициативу при участии регионального бюджета. Однако надо понимать, что возможности федерального и регионального бюджета слишком отличаются. Другой нюанс заключается в диверсификации экономики. Чем больше у региона специализаций, тем более устойчивой является его экономика. На это лишний раз указывает текущий экономический кризис, вызванный резким падением нефтяных цен и остановкой деятельности ряда отраслей из-за пандемии коронавирусной инфекции. В каком положении может оказаться регион, в котором, предположим, две специализации - добыча нефти и туризм? Понятно, что и региону с 27 специализациями будет тяжело в кризис, но его экономика будет функционировать. 
Поэтому целью региональной экономической политики является формирование множества разнообразных специализаций в каждом регионе. Согласно Стратегии, на перспективу до 2025 г. это не планируется, так как в пяти клубах конвергенции (№ 2-6) среднее количество экономических специализаций будет от 5 (клуб 6) до 10 (клуб 3), а по отдельным регионам от 4 (Ненецкий автономный округ) до 12 (Амурская область). При этом в первом клубе среднее количество специализаций равно примерно 20, а по регионам варьирует от 11 (КарачаевоЧеркесская Республика) до 27 (Республика Татарстан, Нижегородская и Ростовская области).

\section{Заключение}

Анализ конвергенции российских регионов по сочетанию перспективных экономических специализаций показал, что цель Стратегии пространственного развития, заключающаяся в сглаживании межрегиональных различий, не может быть достигнута. Различия слишком существенны, чтобы в будущем (к 2025 г.) сходство специализаций регионов допускало максимум 50-процентное отклонение. При заданной мере сходства наиболее непохожие регионы России имеют совпадение перспективных специализаций всего на $8 \%$. Это исключает абсолютную экономическую конвергенцию регионов. Проверка гипотезы о клубной конвергенции позволила идентифицировать шесть групп регионов (клубов). Около 80 \% регионов вошло в первый клуб, характеризующийся наибольшим разнообразием перспективных экономических специализаций. Оставшиеся регионы распределились по пяти клубам в зависимости от сходства небольшого количества специализаций. Отсюда можно констатировать, что экономическое пространство России в отношении перспективных специализаций делится на «центр» (клуб 1) и «периферию» (клубы 2-6). При этом периферийные узкоспециализированные регионы располагаются (кроме Костромской области) вдоль государственной границы Российской Федерации.

Рассмотренный документ пространственного развития содержит ряд неточностей, неопределенностей и отсутствующих разъяснений. При этом была проанализирована только часть Стратегии, касающаяся перспективных экономических специализаций. Гораздо больше вопросов по другим частям, связанным с макрорегионами, перспективными центрами экономического роста, агломерациями и геостратегическими территориями. Некоторые из этих вопросов были поставлены в научных публикациях, но независимая комплексная социально-экономическая экспертиза Стратегии не проводилась. Это необходимо сделать в ближайшей перспективе, поскольку на основе Стратегии планируется разработка документов стратегического планирования в макрорегионах и регионах. Если не проводить такую экспертизу, то концептуальные, эмпирические и другие ошибки, допущенные в Стратегии, сформируют неправильную региональную экономическую политику и, как следствие, не будут способствовать экономическому росту.

\section{Благодарности}

Исследование выполнено за счет средств государственного задания (№ госрегистрации темы АААА-А17-117041910166-3).

\section{Список литературы}

1. Блануца В.И. 2017. Территориальная структура специализации российских регионов на телекоммуникационных услугах. Региональные исследования, (1): 16-24.

2. Блануца В.И. 2018а. Территориальная структура цифровой экономики России: предварительная делимитация «умных» городских агломераций и регионов. Пространственная экономика, (2): 17-35.

3. Блануца В.И. 2018б. Социально-экономическое районирование в эпоху больших данных. М.: ИНФРА-М, 194.

4. Блануца В.И. 2019. Цифровая экономика Сибири: территориальные платформы для кластеров. Актуальные проблемы экономики и права, 13 (3): 1362-1374. 
5. Бухвальд Е.М., Кольчугина А.В. 2019. Стратегия пространственного развития и приоритеты национальной безопасности Российской Федерации. Экономика региона, 15 (3): 631-643.

6. Воронов В.В. 2014. Конвергенция регионов Европейского союза: особенности и оценка. Экономические и социальные перемены: факты, тенденции, прогноз, (6): 85-99.

7. Иванов О.Б., Бухвальд Е.М. 2019. «Перспективная экономическая специализация» как новация политики регионального развития. ЭТАП: экономическая теория, анализ, практика, (6): 49-65.

8. Котов А.В. 2019. Экспортоориентированная сырьевая модель российской экономики: в поисках новой модели пространственного развития. Национальная безопасность и стратегическое планирование, (2): 5-16.

9. Кузнецова О.В. 2019. Стратегия пространственного развития Российской Федерации: иллюзия решений и реальность проблем. Пространственная экономика, 15 (4): 107-125.

10. Лексин В.Н. 2019. Дороги, которые мы не выбираем (о правительственной «Стратегии пространственного развития Российской Федерации на период до 2025 года»). Российский экономический журнал, (3): 3-24.

11. Мельникова Л.В. 2018. Теоретические аргументы и эмпирическое знание в стратегическом планировании. Регион: экономика и социология, (2): 52-80.

12. Минакир П.А. 2019. Российское экономическое пространство: стратегические тупики. Экономика региона, 15 (4): 967-980.

13. Распоряжение Правительства РФ от 28 июля 2017 г. № 1632-р «Об утверждении программы «Цифровая экономика Российской Федерации»»». URL: http://static.government.ru/media/ files/9gFM4FHj4PsB79I5v7yLVuPgu4bvR7M0.pdf (дата обращения: 13 апреля 2020).

14. Распоряжение Правительства РФ от 13 февраля 2019 г. № 207-р «Об утверждении Стратегии пространственного развития до 2025 года». URL: http://static.government.ru/ media/files/UVAlqUtT08o60RktoOX122JjAe7irNxc.pdf (дата обращения: 13 апреля 2020).

15. Смирнова О.О. 2014. Главная государственная «точка роста» России: стратегия пространственного развития Российской Федерации и генеральная схема размещения и развития производительных сил СССР. Методологические основы. Современные производительные силы, (3): 14-21.

16. Смирнова О.П., Аверина Л.М. 2019. Исследование особенностей перспективной экономической специализации индустриального региона. Региональная экономика: теория и практика, 17 (6): 1006-1018.

17. Audretsch D.B., Feldman M.P. 1996. R\&D spillovers and the geography of innovation and production. American Economic Review, 86 (3): 630-640.

18. Balland P.-A., Boschma R., Crespo J., Rigby D.L. 2019. Smart specialization policy in the European Union: Relatedness, knowledge complexity and regional diversification. Regional Studies, 53 (9): $1252-1268$.

19. Barrios C., Flores E., Angeles M.M. 2019. Club convergence in innovation activity across European regions. Papers in Regional Science, 98 (4): 1545-1565.

20. Barro R.J., Sala-i-Martin X. 1992. Convergence. Journal of Political Economy, 100 (2): 223-251.

21. Borsekova K., Vanova A., Vitalisova K. 2017. Smart specialization for smart spatial development: Innovative strategies for building competitive advantages in tourism in Slovakia. SocioEconomic Planning Sciences, 58 (10): 39-50.

22. Celbis M.G., de Crombrugghe D. 2018. Internet infrastructure and regional convergence: Evidence from Turkey. Papers in Regional Science, 97 (2): 387-409.

23. Feder C. 2018. Smart specialization strategy and directed technological change. Economics Bulletin, 38 (3): 1428-1437.

24. Foray D. 2018. Smart specialization strategies and industrial modernization in European regions theory and practice. Cambridge Journal of Economics, 42 (6): 1505-1520.

25. Galor O. 1996. Convergence? Inferences from theoretical models. The Economic Journal, 106 (437): 1056-1069.

26. Gozgor G., Lau C.K.M., Lu Z. 2019. Convergence clustering in the Chinese provinces: New evidence from several macroeconomic indicators. Review of Development Economics, 23 (3): 1331-1346.

27. Humer A. 2018. Linking polycentricity concepts to periphery: Implications for an integrative Austrian strategic spatial planning practice. European Planning Studies, 26 (4): 635-652.

28. Islam N. 2003. What have we learnt from the convergence debate? Journal of Economic Surveys, 17 (3): 309-362.

29. Ivanovski K., Churchill S.A., Smyth R. 2018. A club convergence analysis of per capita energy consumption across Australian regions and sectors. Energy Economics, 76: 519-531. 
30. Kinfemichael B., Morshed A.K.M.M. 2019. Convergence of labor productivity across the US states. Economic Modelling, 76: 270-280.

31. Lopes J., Ferreira J.J., Farinka L. 2019. Innovation strategies for smart specialization (RIS3): Past, present and future research. Growth and Change, 50 (1): 38-68.

32. Marelli E.P., Parisi M.L., Signorelli M. 2019. Economic convergence in the EU and Eurozone. Journal of Economic Studies, 46 (7): 1332-1344.

33. Monfort M., Ordonez J., Sala H. 2018. Inequality and unemployment patterns in Europe: Does integration lead to (real) convergence? Open Economies Review, 29 (4): 703-724.

34. Montalban M., Frigant V., Jullien B. 2019. Platform economy as a new form of capitalism: A Régulationist research programme. Cambridge Journal of Economics, 43 (4): 805-824.

35. Napolitano O., Pietroluongo M., Kounetas K. 2018. Stochastic convergence or divergence of total factor productivity and GDP of Italian Regions. Re-examing the evidence. Economics Bulletin, 38 (4): 18571863.

36. Parr J.B. 1999. Growth-pole strategies in regional economic planning: A retrospective view. Part 1. Origins and advocacy. Urban Studies, 36 (7): 1195-1215.

37. Perroux F. 1950. Economic space: Theory and application. Quarterly Journal of Economics, 64 (1): 89-104.

38. Ranga M. 2018. Smart specialization as a strategy to develop early-stage regional innovation systems. European Planning Studies, 26 (11): 2125-2146.

39. Rios V., Gianmoena L. 2018. Convergence in $\mathrm{CO}_{2}$ emissions: A spatial economic analysis with cross-country interactions. Energy Economics, 75: 222-238.

40. Spulber D.F. 2019. The economics of markets and platforms. Journal of Economics and Management Strategy, 28 (1): 159-172.

41. Tarabar D. 2019. Globalization, cultural distance, and cultural convergence: Some new evidence. Economics Bulletin, 39 (1): 453-466.

42. Tian X., Zhang X., Zhou Y., Yu X. 2016. Regional income inequality in China revisited: A perspective from club convergence. Economic Modelling, 56: 50-58.

43. Von Lyncker K., Thoennessen R. 2017. Regional club convergence in the EU: Evidence from a panel data analysis. Empirical Economics, 52 (2): 525-553.

\section{References}

1. Blanutsa V.I. 2017. The territorial structure of the specialization of the Russian regions in telecommunication services. Regional Studies, (1): 16-24. (in Russian)

2. Blanutsa V.I. 2018a. The territorial structure of the digital economy of Russia: Preliminary delimitation of "smart" urban agglomerations and regions. Spatial Economics, (2): 17-35. (in Russian)

3. Blanutsa V.I. 2018b. Socio-Economic Regionalization in the Era of Big Data. Moscow: INFRA-M Publ., 194. (in Russian)

4. Blanutsa V.I. 2019. Digital economy of Siberia: Territorial platforms for clusters. Actual Problems of Economics and Law, 13 (3): 1362-1374. (in Russian)

5. Bukhvald E.M., Kolchugina A.V. 2019. Spatial development strategy and national security priorities of the Russian Federation. Regional Economics, 15 (3): 631-643. (in Russian)

6. Voronov V.V. 2014. Convergence of the regions of the European Union: features and assessment. Economic and Social Changes: Facts, Trends, Forecast, (6): 85-99. (in Russian)

7. Ivanov O.B., Buchwald E.M. 2019. "Perspective economic specialization" as an innovation in regional development policy. ETAP: Economic Theory, Analysis, Practice, (6): 49-65. (in Russian)

8. Kotov A.V. 2019. Export-oriented raw material model of the Russian economy: In search of a new model of spatial development. National Security and Strategic Planning, (2): 5-16. (in Russian)

9. Kuznetsova O.V. 2019. The spatial development strategy of the Russian Federation: The illusion of solutions and the reality of problems. Spatial Economics, 15 (4): 107-125. (in Russian)

10. Leksin V.N. 2019. Roads that we do not choose (about the government's "Strategy for the spatial development of the Russian Federation for the period until 2025"). Russian Economic Journal, (3): 3-24. (in Russian)

11. Melnikova L.V. 2018. Theoretical arguments and empirical knowledge in strategic planning. Region: Economics and Sociology, (2): 52-80. (in Russian)

12. Minakir P.A. 2019. Russian economic space: Strategic deadlocks. Regional Economics, 15 (4): 967-980. (in Russian) 
13. Decree of the Government of the Russian Federation of July 28, 2017 No. 1632-r "On approval of the program "Digital Economy of the Russian Federation"”. Available at: http://static. government.ru/media/files/9gFM4FHj4PsB79I5v7yLVuPgu4bvR7M0.pdf (accessed April 13, 2020). (in Russian)

14. Decree of the Government of the Russian Federation of February 13, 2019 No. 207-r "On approval of the Strategy for spatial development until 2025". Available at: http://static. government.ru/media/files/UVAlqUtT08o60RktoOX122JjAe7irNxc.pdf (accessed April 13, 2020). (in Russian)

15. Smirnova O.O. 2014. The main state "growth point" of Russia: The spatial development strategy of the Russian Federation and the general layout of the deployment and development of the productive forces of the USSR. Methodological basis. Modern Productive Forces, (3): 14-21. (in Russian)

16. Smirnova O.P., Averina L.M. 2019. A study of the features of the promising economic specialization of the industrial region. Regional Economics: Theory and Practice, 17 (6): 1006-1018. (in Russian)

17.Audretsch D.B., Feldman M.P. 1996. R\&D spillovers and the geography of innovation and production. American Economic Review, 86 (3): 630-640.

18.Balland P.-A., Boschma R., Crespo J., Rigby D.L. 2019. Smart specialization policy in the European Union: Relatedness, knowledge complexity and regional diversification. Regional Studies, 53 (9): $1252-1268$.

19.Barrios C., Flores E., Angeles M.M. 2019. Club convergence in innovation activity across European regions. Papers in Regional Science, 98 (4): 1545-1565.

20.Barro R.J., Sala-i-Martin X. 1992. Convergence. Journal of Political Economy, 100 (2): 223-251.

21.Borsekova K., Vanova A., Vitalisova K. 2017. Smart specialization for smart spatial development: Innovative strategies for building competitive advantages in tourism in Slovakia. Socio-Economic Planning Sciences, 58 (10): 39-50.

22. Celbis M.G., de Crombrugghe D. 2018. Internet infrastructure and regional convergence: Evidence from Turkey. Papers in Regional Science, 97 (2): 387-409.

23. Feder C. 2018. Smart specialization strategy and directed technological change. Economics Bulletin, 38 (3): 1428-1437.

24. Foray D. 2018. Smart specialization strategies and industrial modernization in European regions - theory and practice. Cambridge Journal of Economics, 42 (6): 1505-1520.

25. Galor O. 1996. Convergence? Inferences from theoretical models. The Economic Journal, 106 (437): 1056-1069.

26. Gozgor G., Lau C.K.M., Lu Z. 2019. Convergence clustering in the Chinese provinces: New evidence from several macroeconomic indicators. Review of Development Economics, 23 (3): 1331-1346.

27. Humer A. 2018. Linking polycentricity concepts to periphery: Implications for an integrative Austrian strategic spatial planning practice. European Planning Studies, 26 (4): 635-652.

28. Islam N. 2003. What have we learnt from the convergence debate? Journal of Economic Surveys, 17 (3): 309-362.

29. Ivanovski K., Churchill S.A., Smyth R. 2018. A club convergence analysis of per capita energy consumption across Australian regions and sectors. Energy Economics, 76: 519-531.

30. Kinfemichael B., Morshed A.K.M.M. 2019. Convergence of labor productivity across the US states. Economic Modelling, 76: 270-280.

31. Lopes J., Ferreira J.J., Farinka L. 2019. Innovation strategies for smart specialization (RIS3): Past, present and future research. Growth and Change, 50 (1): 38-68.

32. Marelli E.P., Parisi M.L., Signorelli M. 2019. Economic convergence in the EU and Eurozone. Journal of Economic Studies, 46 (7): 1332-1344.

33. Monfort M., Ordonez J., Sala H. 2018. Inequality and unemployment patterns in Europe: Does integration lead to (real) convergence? Open Economies Review, 29 (4): 703-724.

34. Montalban M., Frigant V., Jullien B. 2019. Platform economy as a new form of capitalism : A Régulationist research programme. Cambridge Journal of Economics, 43 (4): 805-824.

35. Napolitano O., Pietroluongo M., Kounetas K. 2018. Stochastic convergence or divergence of total factor productivity and GDP of Italian Regions. Re-examing the evidence. Economics Bulletin, 38 (4): 18571863.

36. Parr J.B. 1999. Growth-pole strategies in regional economic planning: A retrospective view. Part 1. Origins and advocacy. Urban Studies, 36 (7): 1195-1215. 
37. Perroux F. 1950. Economic space: Theory and application. Quarterly Journal of Economics, 64 (1): 89-104.

38. Ranga M. 2018. Smart specialization as a strategy to develop early-stage regional innovation systems. European Planning Studies, 26 (11): 2125-2146.

39. Rios V., Gianmoena L. 2018. Convergence in $\mathrm{CO}_{2}$ emissions: A spatial economic analysis with cross-country interactions. Energy Economics, 75: 222-238.

40. Spulber D.F. 2019. The economics of markets and platforms. Journal of Economics and Management Strategy, 28 (1): 159-172.

41. Tarabar D. 2019. Globalization, cultural distance, and cultural convergence: Some new evidence. Economics Bulletin, 39 (1): 453-466.

42. Tian X., Zhang X., Zhou Y., Yu X. 2016. Regional income inequality in China revisited: A perspective from club convergence. Economic Modelling, 56: 50-58.

43. Von Lyncker K., Thoennessen R. 2017. Regional club convergence in the EU: Evidence from a panel data analysis. Empirical Economics, 52 (2): 525-553.

\section{Ссылка для цитирования статьи For citation}

Блануца В.И. 2020. Перспективные экономические специализации для российских регионов в Стратегии пространственного развития: клубы конвергенции. Экономика. Информатика. 47 (2): 233 243. DOI: 10.18413/2687-0932-2020-47-2-233-243.

Blanutsa V.I. 2020. Perspective economic specializations for the russian regions in the Strategy of spatial development: convergence clubs. Economics. Information technologies. 47 (2): 233-243 (in Russian). DOI: $10.18413 / 2687-0932-2020-47-2-233-243$. 